\title{
Impact of Training Images on Radiometric Compensation
}

Vignesh Sankar, Ahmed Gawish, Paul Fieguth, Lamm Mark
University of Waterloo, ON, Canada

University of Waterloo, ON, Canada

University of Waterloo, ON, Canada

Christie Digital Systems, Kitchener, ON, Canada

\section{Abstract}

The increasing availability of both high-resolution projectors and imperfect displays make radiometric correction an essential component in all modern projection systems. Particularly, projecting in casual locations, such as classrooms, open areas and homes, calls for the development of radiometric correction techniques that are fully automatic and deal with display imperfections in real-time. This paper reviews the current radiometric compensation algorithms and discusses the influence of different training images on their performance.

\section{Introduction}

Radiometric compensation is the process of changing projector images such that visual quality of the image is maintained when projected on a textured background [1][2][3]. This is accomplished by a projector - camera (pro-cam) system, where the camera provides the feedback of projection on the surface, which is then used to compensate for a desired image.

Pro-cam system calibration is a prerequisite for Radiometric Compensation (RC). Geometric calibration provides the pixel correspondence between the projector and camera. Structured light patterns are projected and captured by the camera to acquire the required calibration. After the system is geometrically calibrated, radiometric functions are used to model the transformation from the projector pixel intensity to its corresponding camera pixel intensity [1][2][3]. The training set for the radiometric model development is obtained by projecting colored images and capturing their response from the screen. Linear $\mathrm{RC}$ models require the camera to be calibrated in order to remove the nonlinear response of the camera [1]. A summary of the existing methods to calculate the camera response function can be found in [4].

Bimber et al. [3] proposed a multi-projector approach where each channel is compensated independently. However, as the spectral responses of the projector and camera can overlap, $\mathrm{Na}$ yar et al. [1] proposed a $3 \times 3$ matrix to model the transformation between the projector and camera.

$$
C=V P
$$

where, $C$ and $P$ are the camera and projector image pixels respectively and $V$ is the color mixing matrix, which models the surface reflectance property of the screen and the overlap of spectral response between the projector and camera. It is a pixel level model, where the color mixing matrix is calculated for each projector- camera pixel pair. Yoshiha et al. [2] extended the linear model to $3 \times 4$ matrix by considering environmental light and projector black level. Grossberg et al. [5] proposed an algorithm which uses a minimal set of 6 images to find the extended color mixing matrix and projector response function.

Mihara et al. [6] proposed to use high spatial resolution camera to capture the steep reflection variation across a single projector pixel. In this method, multiple camera pixels are used to capture the image of one projector pixel and a linear model is developed for each camera pixel. Grundhofer et al. [7] proposed a thin plate splines interpolation with radial basis function to model the radiometric compensation function. The training images are formed by the spare sampling of projector gamut. Grundhofer's nonlinear model does not require the camera to be pre-calibrated.

The aforementioned RC methods assume a pixel level model, where each camera pixel intensity is a function of only one projector pixel intensity. Also, the environmental light is considered to be constant. Camera should be pre-calibrated for the linear model, whereas the nonlinear model assumes that the camera can capture the entire projector gamut without saturation.

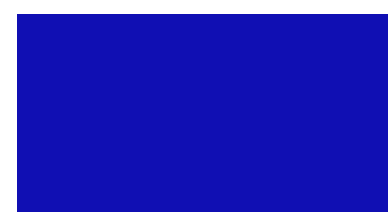

Projector image

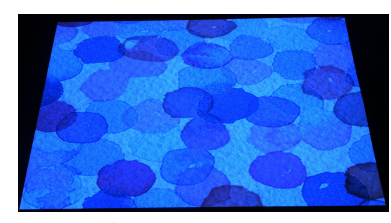

Camera image
Fig. 1: Sample images in plain training set.

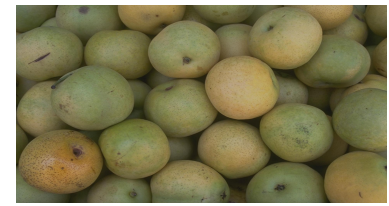

Projector image

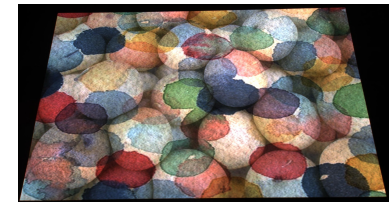

Camera image
Fig. 2: Sample images in textured training set.

\section{Analysis of Training images}

Linear and nonlinear radiometric approaches are used to model the transformation between the camera and projector images. A training set plays a very important role in building a robust radiometric model. Hence, the purpose of this paper is to discuss the impact of different training images on the performance of the radiometric approaches.

The Modular Transfer Function (MTF) of the imaging system is its ability to transfer the contrast of the object to the image. The function models the reduction in contrast of the object while going through an imaging system as a function of spatial resolution of the contrast [8]. Since both the projector and camera have MTF, the pro-cam system's MTF is the product of its individual MTF. Hence, when an image $P$ is projected and captured by the camera, the camera image $C$ contains a contrast reduced version of $P$. The amount of contrast reduction depends on the magnitude and spatial frequency of the contrast. This results in a spatially dependent pro-cam system.

Spatial dependency can also occur because of inter pixel coupling [9], which includes leakage of projector brightness to adjacent pixels or activation of a camera sensor by multiple projector pixels. Such inaccuracies in pro-cam devices can make the captured camera pixel intensity depend on multiple projector pixel intensity. Also, these inaccuracies are prominent in the regions of contrast difference [9]. The camera pixels on the border of the darker side of contrast difference are influenced by the brighter side and vice versa. This leads to contrast reduction in the camera image.

MTF and inter pixel coupling are the two major reasons behind the contrast reduction of the object in its image. Both the projector and camera suffer from the above mentioned defects. The resultant spatial dependency from MTF and inter pixel coupling contradicts the primary assumption made in the existing $\mathrm{RC}$ algorithms. As mentioned earlier, both the linear and nonlinear algorithms assume a pixel level model where the camera pixel is affected by only one projector pixel. Since we are modeling a spatially dependent system with a spatially independent model, using different training images will have different results for a RC algorithm.

Four scenarios are explored to understand the dependency of radiometric approach on training images:

\section{- Plain training set with textured background}

- Textured training set with textured background 


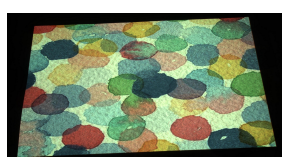

Textured Background
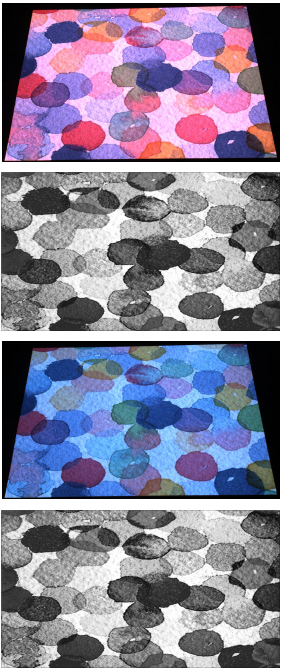

(a) Direct Projection

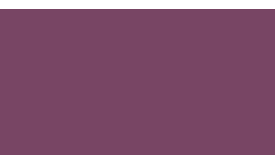

Image1

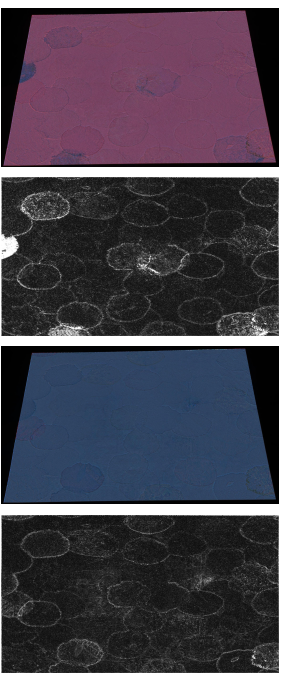

(b) Textured training set

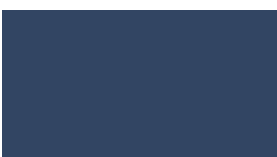

Image2
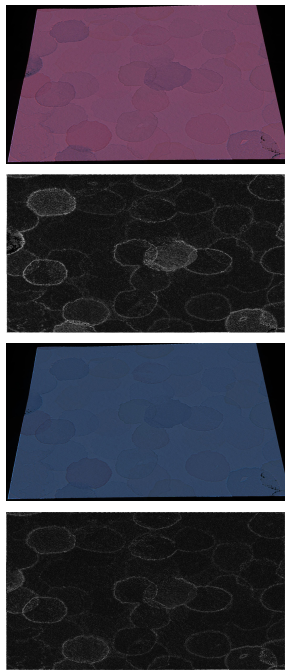

(c) plain training set

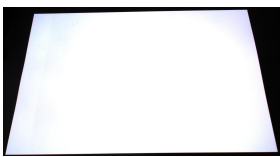

Plain Background
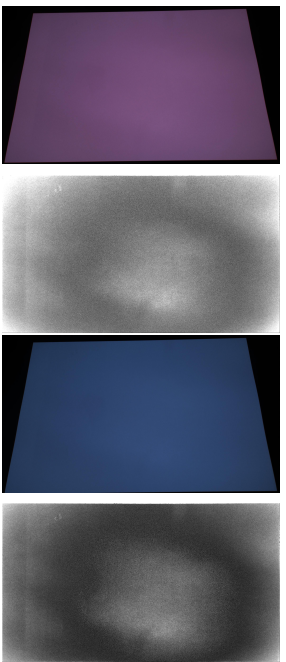

(d) Direct Projection

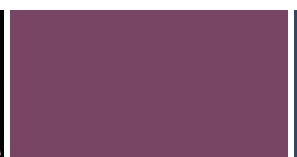

Image1
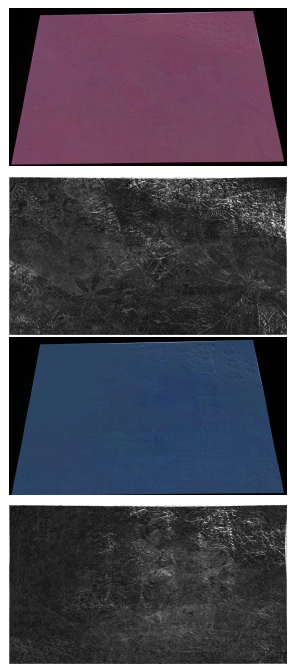

(e) Textured training set

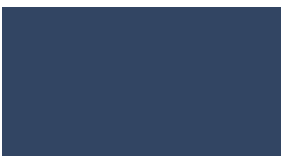

Image2
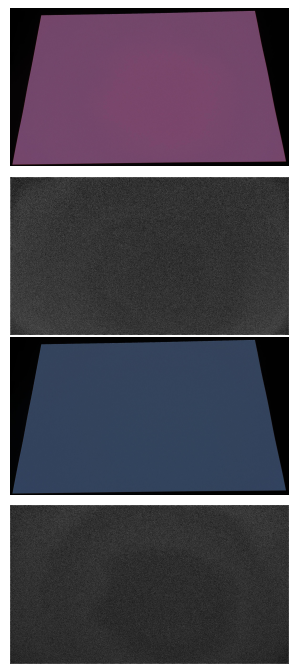

(f) plain training set

Fig. 3: Comparison between direct projection and compensation by the linear model trained with textured and plain training sets. Columns (a), (b) \& (c) show the results with textured background, while (d), (e) \& (f) show the results with plain background surface. Second and third row shows the RMSE images which is the error map between the target image and the compensated image. The error maps are enhanced for visualization. The plain training set performs better then the textured training set for both backgrounds. The textured training set introduces additional errors because of the contrast present in the projector images.

RMSE

\begin{tabular}{|c|c|c|c|}
\hline Image & Direct & Textured & Plain \\
\hline Image1-Background1 & 17.55 & 5.45 & 4.18 \\
\hline Image2-Background1 & 19.65 & 6.99 & 5.07 \\
\hline Image1-Background2 & 8.85 & 4.23 & 2.54 \\
\hline Image2-Background2 & 10.49 & 4.56 & 3.72 \\
\hline
\end{tabular}

CIE2000

\begin{tabular}{|c|c|c|c|}
\hline Image & Direct & Textured & Plain \\
\hline Image1-Background1 & 10.44 & 1.70 & 1.51 \\
\hline Image2-Background1 & 12.69 & 2.04 & 1.71 \\
\hline Image1-Background2 & 5.58 & 1.52 & 0.98 \\
\hline Image2-Background2 & 6.49 & 1.61 & 1.24 \\
\hline
\end{tabular}

SSIM

\begin{tabular}{|c|c|c|c|}
\hline Image & Direct & Textured & Plain \\
\hline Image1-Background1 & 0.552 & 0.982 & 0.988 \\
\hline Image2-Background1 & 0.507 & 0.965 & 0.982 \\
\hline Image1-Background2 & 0.930 & 0.989 & 0.992 \\
\hline Image2-Background2 & 0.931 & 0.984 & 0.990 \\
\hline
\end{tabular}

Table 1: RMSE, CIE2000 and SSIM scores for images. Direct represents Projection without compensation, Textured and Plain represents compensation using textured and plain training sets respectively, RMSE and CIE2000, smaller is better, whereas SSIM larger is better.

- Textured training set with plain background

- Plain training set with plain background

Figure 3(a) \& (d) shows textured and plain backgrounds respectively. Colored projector images without contrast are projected and captured by the camera to form the plain training set as shown in Figure 1. Here, the projector images doesn't contain contrast difference, whereas the camera images contain the contrast difference of the background. The textured training set is composed of real world images as projector images and their corresponding camera images as shown in Figure 2. Textured training set with plain background is used to study the artifacts produced by training images without interference from the background.

The errors resulting from $\mathrm{RC}$ algorithms can be classified into two broad categories, namely radiometric error and saturation error. The radiometric error is caused by the inability of the $\mathrm{RC}$ algorithm to reproduce the target intensity in the resultant camera image. This can be due to the device inaccuracies or assumptions in the RC algorithm. The saturation error is caused by the limited dynamic range of the projector, which occurs when the required projection intensity given by the $\mathrm{RC}$ algorithm exceeds the projector gamut. The following section discusses the radiometric error caused by using different training images.

\section{Results and Conclusion}

Single chip DLP projector at $1920 \times 1200$ resolution along with 5MP gigabit ethernet machine vision camera from Point Grey are used to form the pro-cam system. Device specific artifacts are avoided by repeating the experiments with different $5 \mathrm{MP}$ ethernet machine vision cameras. The Gray scale coding algorithm [10] is used to acquire the geometric calibration between the projector and camera. The textured background shown in Figure 1, is printed on paper and pasted against the wall to form a flat surface.

The grossberg's linear model is implemented in Matlab to show the impact of different training images on RC algorithms. International Commission on Illumination color difference metric $\Delta E_{00}^{*}$ (CIE2000) [11] along with Root Mean Square Error (RMSE) and Structural Similarity Index (SSIM) [12] are used as evaluation metrics for image comparison. CIE2000 score provides the perceived difference between two colors by the human eye. The score increases with higher perceptual difference. In CIE2000, the images are converted into a device independent $\mathrm{CIE} \mathrm{L}^{*} \mathrm{a}^{*} \mathrm{~b}^{*}$ color space and the score is calculated by incorporating the spatial, hue and lightness characteristic of the human eye. RMSE provides the absolute difference between the two colors. RMSE scores helps in identifying the existing errors, whereas CIE2000 helps in identifying errors that are significant to the observers. Lower scores by CIE2000 and RMSE, and higher scores by SSIM metrics indicate better performance.

The results of the experiments are illustrated in Figure 3. Columns (d), (e) and (f) show the results of compensation on plain background surface (Background-2). Second and third row show the RMSE error map between the target image and the compensated image. It helps in identifying the regions of artifacts in the compensated image. From the RMSE images we can see that using textured training set creates more artifacts than using plain training set as shown in figure $3(\mathrm{e})$. The error pattern for textured training set is common for both image 1 and image2. The pattern depicts an 
amalgamation of the contrast present in the training images shown in Figure 4. As discussed earlier, the camera captures the contrast reduced version of the scene. The $\mathrm{RC}$ algorithm attributes this contrast reduction of the projector images to the background texture and overcomes it by increasing the brightness in the regions of contrast. This increased brightness causes artifacts in the compensated image. Since the camera images of the plain training set does not contain any contrast, the camera accurately captures the reflectance properties of the background and is able to produce the desired image on the screen

Similar observations are drawn from the results of background1. We can see that the plain training set is not able to hide the background texture. The linear model delivers satisfactory results in the uniform regions, but deteriorates from the target in the regions of contrast difference of the background. This phenomenon can be clearly observed in the RMSE images. The camera images of the plain training set contain the contrast of the background surface. Inaccurate reading of this contrast by the camera results in artifacts in the compensated images as shown in figure 3(c). The camera images of the textured training set contain contrast of both the background surface and projector images. Hence, RMSE images of the textured training set contain artifacts from both these contrasts.

The saturated pixel locations of the projector image are omitted during the calculation of results. A pixel location is considered to be saturated when at least one channel value goes beyond the projector gamut (0-255). Omission of these pixel locations helps us to understand the radiometric error without the influence of saturation. Table 1 gives the RMSE, CIE2000 and SSIM scores for the images in figure 3. From the table, we can observe that the plain training set performs better then the textured training set. The best results are given by using the plain training set with the plain background. Using textured training set with textured background provides the least performance.

This paper explored the impact of different training images on the performance of the linear RC algorithm with two backgrounds. The results of compensation using the plain and textured training set with the plain and textured background are discussed in detail. It is observed that the performance of the $\mathrm{RC}$ algorithm is enhanced by reducing the contrast of the scene in the camera images of the training set. The reduced contrast minimizes the MTF and inter pixel coupling errors of the camera. Plain training set performs better than the textured training set as the contrast of the scene in plain training set is less compared to textured training set. Similarly, the results of compensation with the plain background are better than the textured background. Future work involves proposing a new procedure to acquire the training images that can assist in the development of radiometric compensation algorithms.

\section{Acknowledgments}

We would like to thank Ontario Centres of Excellence - Voucher for Innovation and Productivity II (OCE-VIP II), The Natural Sciences and Engineering Research Council of Canada - Collaborative Research and Development (NSERC-CRD) and Christie Digital Systems for sponsoring this research work.

\section{References}

[1] S. K. Nayar, H. Peri, M. D. Grossberg, and P. N. Belhumeur, "A projection system with radiometric compensation for screen imperfections," in ICCV workshop on projector-camera systems (PROCAMS), vol. 3. Citeseer, 2003.

[2] T. Yoshida, C. Horii, and K. Sato, "A virtual color reconstruction system for real heritage with light projection," in Proceedings of VSMM, vol. 3, 2003.

[3] O. Bimber, A. Emmerling, and T. Klemmer, "Embedded entertainment with smart projectors," Computer, vol. 38, no. 1, pp. 48-55, 2005.

[4] Y.-W. Tai, X. Chen, S. Kim, S. J. Kim, F. Li, J. Yang, J. Yu, Y. Matsushita, and M. S. Brown, "Nonlinear camera response

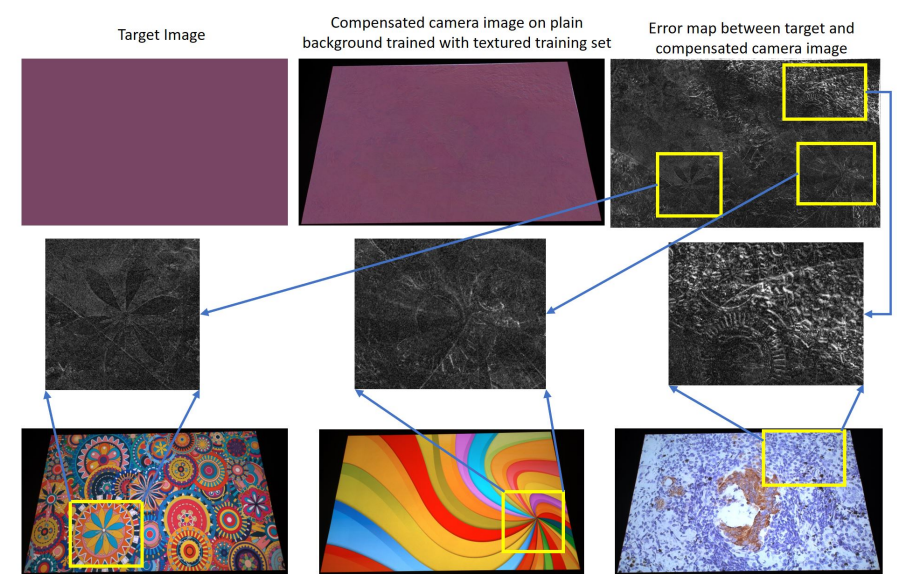

Fig. 4: Artifacts caused by the textured training set. First row shows the target image, compensated camera image and error map. Second row shows magnified regions of the error map. Third row shows the sample of the camera images present in the textured training set. It is observed that the contrast of the camera images in the training set (third row) is being reflected as artifacts in the error map

functions and image deblurring: Theoretical analysis and practice," IEEE transactions on pattern analysis and machine intelligence, vol. 35, no. 10, pp. 2498-2512, 2013.

[5] M. D. Grossberg, H. Peri, S. K. Nayar, and P. N. Belhumeur, "Making one object look like another: Controlling appearance using a projector-camera system," in Computer Vision and Pattern Recognition, 2004. CVPR 2004. Proceedings of the 2004 IEEE Computer Society Conference on, vol. 1. IEEE, 2004, pp. I-I.

[6] S. Mihara, D. Iwai, and K. Sato, "Artifact reduction in radiometric compensation of projector-camera systems for steep reflectance variations," vol. 24 , no. 9 . IEEE, 2014, pp. 16311638.

[7] A. Grundhöfer and D. Iwai, "Robust, error-tolerant photometric projector compensation," vol. 24, no. 12 . IEEE, 2015, pp. 5086-5099.

[8] C. S. Williams and O. A. Becklund, "Introduction to the transfer function." SPIE- The International society for optical engineering.

[9] J.-S. Liu and H. H. Chen, "Preserving image color appearance on non-white projection surfaces," in Multimedia \& Expo Workshops (ICMEW), 2015 IEEE International Conference on. IEEE, 2015, pp. 1-6.

[10] S. Inokuchi, "Range-imaging system for 3d object recognition," in Proc. of 7th International Conference on Pattern Recognition, 1984, 1984.

[11] G. Sharma, W. Wu, and E. N. Dalal, "The ciede2000 colordifference formula: Implementation notes, supplementary test data, and mathematical observations," Color Research \& Application, vol. 30, no. 1, pp. 21-30, 2005.

[12] Z. Wang, A. C. Bovik, H. R. Sheikh, and E. P. Simoncelli, "Image quality assessment: from error visibility to structural similarity," IEEE transactions on image processing, vol. 13, no. 4, pp. 600-612, 2004. 